\title{
A feasibility study of sequential paclitaxel and S-1 (PTX/S-1) chemotherapy as postoperative adjuvant chemotherapy for advanced gastric cancer
}

\author{
Michiya Kobayashi ${ }^{1}$, Akira Tsuburaya ${ }^{2}$, NaOki Nagata $^{3}$, Yumi Miyashita ${ }^{4}$, Koji Oba ${ }^{5}$, and Junichi Sakamoto $^{5}$ \\ ${ }^{1}$ Department of Tumor Surgery, Kochi Medical School, Kohasu, Oko-cho, Nankoku 783-8505, Japan \\ ${ }^{2}$ Department of Surgery, Kanagawa Cancer Center, Yokohama, Japan \\ ${ }^{3}$ First Department of Surgery, University of Occupational and Environmental Health, Kitakyushu, Japan \\ ${ }^{4}$ Department of Clinical Research, Aichi Cancer Center, Aichi Hospital, Okazaki, Japan \\ ${ }^{5}$ Department of Epidemiological and Clinical Research Information Management, Kyoto University, Graduate School of Medicine, Kyoto, \\ Japan
}

\begin{abstract}
Background. The most frequent recurrence pattern of advanced gastric cancer is peritoneal dissemination. We investigated the safety of and compliance with sequential chemotherapy consisting of paclitaxel and $S-1$, both of which are effective in the treatment of peritoneal dissemination.

Methods. The patients in the study all had histologically proven gastric cancer, classified according to the TNM and the Japanese criteria for gastric cancer as T3-4, N0-2, P0, H0 M0, and CY0-1. In all patients, standard gastrectomy of more than a $\mathrm{D2}$ dissection was performed. A dose of $80 \mathrm{mg} / \mathrm{m}^{2}$ of paclitaxel was administered for three courses. One course comprised weekly administration for 3 weeks, followed by a 1week rest, except for the first course (following S-1 administration at $80 \mathrm{mg} / \mathrm{m}^{2}$ body surface area), in which paclitaxel was administered for only 2 weeks, followed by a 1-week rest. S-1 was administered from day 78 for four courses, with one course comprising 2 weeks' administration followed by a 1week rest. Fifty patients received paclitaxel chemotherapy. The median age was 62.5 years overall; among the 34 male patients it was 65.5 years, and among the female patients it was 48.0 years.

Results. The patient compliance rate was $84 \%$. There were no cases of grade 4 hematological toxicity during either paclitaxel or S-1 treatment. With respect to nonhematological toxicities, there was one case of grade 3 neuropathy during the course of paclitaxel treatment and one case of grade 3 diarrhea during the course of S-1 treatment. These patients recovered and completed the scheduled treatment regimen.

Conclusion. Sequential chemotherapy of paclitaxel and S-1 as postoperative adjuvant chemotherapy for advanced gastric cancer is feasible.
\end{abstract}

Key words Gastric cancer · Chemotherapy · Paclitaxel · S-1 · Adjuvant chemotherapy

Offprint requests to: $\mathrm{M}$. Kobayashi

Received: November 18, 2005 / Accepted: January 27, 2006

\section{Introduction}

Although the surgical treatment of advanced gastric cancer has progressed, about $50 \%$ of patients with serosal involvement develop peritoneal recurrence, despite receiving $\mathrm{R} 0$ resection [1-4].

In Japan, oral fluoropyrimidine agents have historically been used for adjuvant chemotherapy for gastric cancer. Of these agents, UFT, a combination of tegafur and uracil, has been one of the most popular. A recently developed drug, known as S-1, is a novel oral fluoropyrimidine agent - containing 5-chloro-2,4dihydroxypyridine and oxonic acid - that exploits the biochemical modulatory activity of 5-fluorouracil. There are several reports that S-1 is effective for peritoneal dissemination after curative resection of advanced gastric cancer [5-8].

Paclitaxel (PTX) is a potent inhibitor of cell replication and was originally isolated from the bark of the tree Taxus brevifolia. The antimitotic activity of PTX results from an effective blocking of the late $\mathrm{G} 2$ and $\mathrm{M}$ phases in the cell cycle. Several reports have demonstrated that systemic administration of PTX is effective in the control of malignant ascites in gastric cancer patients $[9,10]$.

Combination therapy using S-1 and PTX for the prevention of peritoneal recurrence of gastric cancer is theoretically promising, due to the unique inhibitory activities of these drugs. As part of our investigation into the clinical applicability of combination therapy using S- 1 and PTX, we are conducting a factorial design study of oral fluorouracil agents, such as UFT and S-1, versus combinations of these agents with PTX (SAMIT trial) [11].

In the present study we investigated the feasibility of combination chemotherapy using S-1 and PTX, shown to be the most toxic combination to patient of the four combinations investigated in the above-mentioned 
large-scale clinical trial. The endpoints of interest in the present study were the time scale of the treatment regimen and the rate of occurrence of severe adverse events.

\section{Patients and methods}

\section{Eligibility}

Patients in this study had histologically proven gastric cancer. That is, they showed T3-4 (T3, tumor penetration of serosa; T4, tumor invasion of adjacent structures), N0-2 (no lymph node metastasis or lymph node metastasis to group 1 or 2), P0 (no peritoneal metastasis), H0 (no liver metastasis), M0 (no other distant metastasis), and CY0-1 (benign/indeterminate cells on peritoneal cytology), according to the TNM and the Japanese criteria for gastric cancer. Standard gastrectomy with more than a D2 dissection was performed in all patients. Patients were registered from 2 to 8 weeks following the operation, and chemotherapy commenced within 1 week of registration when it was clear that no other active cancer was present in other organs. Other inclusion criteria were: age 20 to 80 years, Eastern Cooperative Oncology Group (ECOG) performance status [12] 0 or 1 , no history of previous chemotherapy or radiotherapy, adequate bone marrow function (4000/ $\mathrm{mm}^{3}<$ white cell count $<12000 / \mathrm{mm}^{3}$ or $2000 / \mathrm{mm}^{3}<$ neutrophil count $<6000 / \mathrm{mm}^{3}$; platelet count $>10000 / \mathrm{mm}^{3}$; and hemoglobin $>8.0 \mathrm{~g} / \mathrm{dl}$ ), adequate liver function (bilirubin level $<1.5 \mathrm{mg} / \mathrm{dl}$, aspartate aminotransferase [AST] or alanine aminotransferase [ALT] $>100 \mathrm{IU} / 1$ or less than twice the upper limit of normal), adequate renal function (creatinine $<1.5 \mathrm{mg} / \mathrm{dl}$, and creatinine clearance $>50 \mathrm{ml} / \mathrm{min}$ ). All patients gave written informed consent, conforming to institutional guidelines, indicating that they were aware of the investigational nature of the study. This protocol was approved by the ethics committees of all participating institutions.

\section{Treatment}

Sequential chemotherapy, of PTX following S-1 administration was performed in all patients. PTX was administered for a total of 8 weeks. A dose of PTX of $80 \mathrm{mg}$ per $\mathrm{m}^{2}$ body surface area of the patient was given, by a 1-h drip infusion after administering standard prophylaxis for taxol-associated hypersensitivity reactions; i.e., dexamethasone (20 mg i.v.), diphenhydramine (25 mg p.o.), and ranitidine (50 mg i.v.). PTX was administered for three courses following S-1 administration, with one course comprising weekly administration for 3 weeks then 1-week rest, except for the first course, in which PTX was administered for only 2 weeks, followed by a 1-week rest. S-1 was administered orally from day 78, at $80 \mathrm{mg}$ per $\mathrm{m}^{2}$ body surface area, for four courses. Each course comprised 2 weeks' administration followed by a 1-week rest.

\section{Patient evaluation and follow-up}

Upon entry into the study, all patients completed a medical history and underwent a physical examination in addition to laboratory studies (complete blood cell count, urinalysis, electrolytes, and renal and liver function tests), chest X-ray, and electrocardiogram. Computed tomography (CT), plain radiograms, and other appropriate imaging tests were performed. Complete blood cell counts, urinalysis, electrolytes, and renal and liver function tests were evaluated at least once weekly, before subsequent cycles, and at the end of protracted administration of PXT and S-1. CT was performed at the start of the third course of PTX treatment and at the start of the fourth course of S-1 treatment.

\section{Toxicity}

Toxicity was monitored and graded according to the third version of the National Cancer Institute Common Toxicity Criteria (NCI-CTC) [13]. Assessment of nonhematological toxicity was performed throughout treatment. The correlation between the appearance of severe grade $3 / 4$ toxicity and compliance with the chemotherapy schedule (postponement, stopping, dose decrease) was also investigated.

\section{Statistical methods}

This study was designed to evaluate 50 postoperative patients with advanced gastric cancer. The sample size was calculated on the basis that the rate for complete accomplishment of the schedule was expected to range from $75 \%$ to $85 \%$. Given the sample size, $95 \%$ twosided confidence intervals for this rate were estimated as $10 \%$ to $12 \%$, using the normal approximation of binomial variables.

\section{Results}

Fifty-one patients were registered in this study. One patient withdrew agreement prior to the start of the chemotherapy. Fifty patients received PTX chemotherapy. Table 1 shows the patients' characteristics. The median age was 62.5 years overall, among the 34 male patients it was 65.5 years, and among the female patients it was 48.0 years. Most of the patients had a performance status of 0 . The number of patients in categories T2, T3, and T4 were 1, 44, and 5, respectively. 
Eight patients had no lymph node metastasis. The numbers of patients with lymph node metastasis to stations N1, N2, and N3 were 20, 21, and 1, respectively. According to the Japanese criteria for gastric cancer, 12, 15, 16 , and 7 patients were at stages $2,3 \mathrm{a}, 3 \mathrm{~b}$, and 4 , respectively.

Eight patients did not accomplish the planned treatment schedule; 5 due to toxicity, 2 due to agreement withdrawal, and 1 due to disease progression. Forty-two patients accomplished the full schedule of the treatment regimen (Fig. 1). Table 2 shows patient compliance with the PTX/S-1 sequential chemotherapy. The rate of total accomplishment of the schedule (i.e., patient compliance) was $84 \%$. Table 3 lists the numbers of patients in whom the dose was decreased $\left(-10 \mathrm{mg} / \mathrm{m}^{2}\right)$ or in whom the treatment schedule was postponed due to toxicity. The most common toxicity was neutropenia. Two patients caught colds, and PTX administration was postponed. In 1 patient, the dose was decreased due to grade 3 diarrhea during the second course of S- 1 treatment. Table 4 shows the hematological toxicities. Leucopenia, neutropenia, thrombocytopenia, and anemia were observed in $30(60 \%), 29(58 \%), 3(6 \%)$, and 40 $(80 \%)$ patients, respectively, during PTX administra-

Table 1. Patients' characteristics

\begin{tabular}{llc}
\hline Age, years & Male $(n=34)$ & $62.5(34-74)^{\mathrm{a}}$ \\
& Female $(n=16)$ & $48.0(35-70)^{\mathrm{a}}$ \\
PS & 0 & 42 \\
& 1 & 5 \\
pT & 2 & 1 \\
& 3 & 44 \\
$\mathrm{~N}$ & 4 & 5 \\
& 0 & 8 \\
& 1 & 20 \\
Stage & 2 & 21 \\
& 3 & 1 \\
& 2 & 12 \\
& $3 \mathrm{a}$ & 15 \\
& $3 \mathrm{~b}$ & 16 \\
\hline
\end{tabular}

PS, performance status [12]

${ }^{a}$ Range tion. Grade 3 toxicities included four cases of neutropenia and one case of leucocytopenia. No patients had fever. The above hematological toxicities during S-1 administration were observed in 19 (41.3\%), 26 (59.1\%), 9 (20.5\%), and $39(88.6 \%)$ patients, respec-
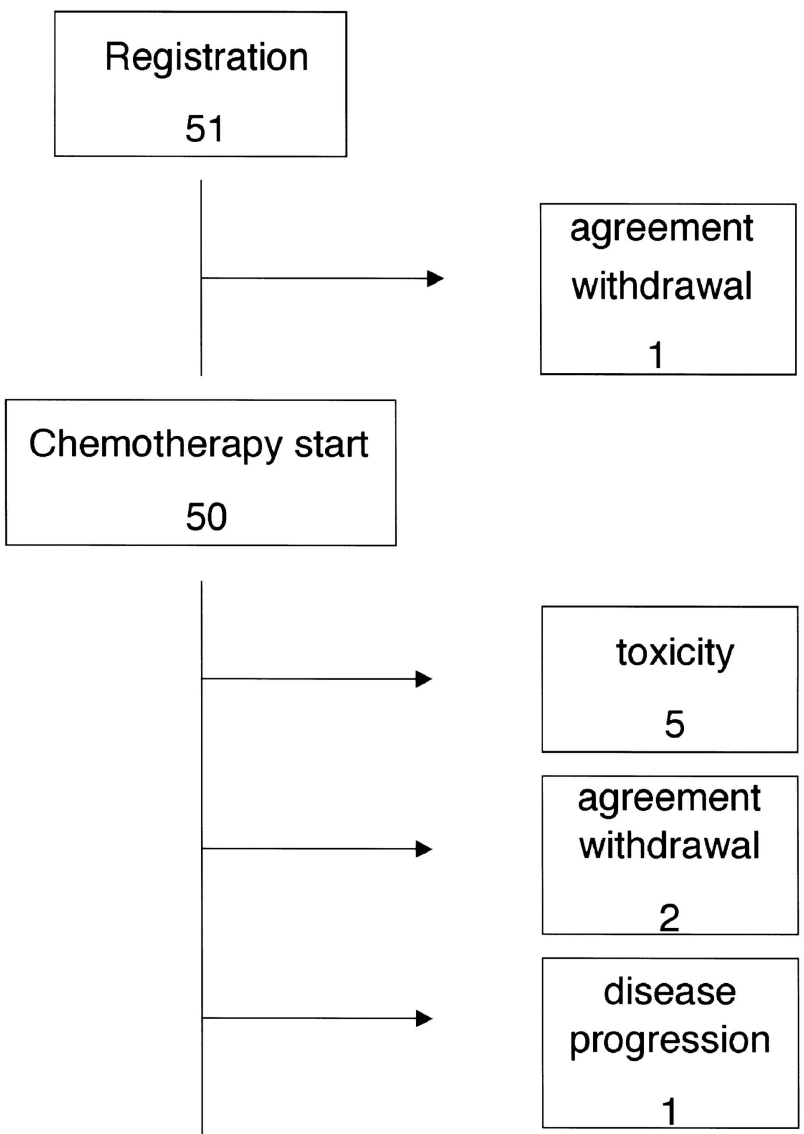

\section{Accomplishment of schedule 42}

Fig. 1. Numbers of patients according to accomplishment of the treatment regimen schedule

Table 2. Patient compliance with PTX/S-1 sequential chemotherapy after radical surgery

\begin{tabular}{lcccl}
\hline & $\begin{array}{c}\text { Number of courses } \\
\text { accomplished }\end{array}$ & $\begin{array}{c}\text { Number of } \\
\text { dropouts }\end{array}$ & $\begin{array}{c}\text { Compliance } \\
(\%)\end{array}$ & \multicolumn{1}{c}{ Cause } \\
\hline & 0 & 1 & 98 & Agreement withdrawal \\
PTX & 1 & 3 & 92 & Toxicity (neutropenia, 2; allergy, 1) \\
PTX & 2 & 1 & 90 & Agreement withdrawal \\
PTX & 3 & 1 & 88 & Disease progression \\
S-1 & 4 & 1 & 86 & Toxicity (dyspnea) \\
S-1 & 5 & 1 & 86 & Toxicity (neutropenia) \\
S-1 & 6 & 0 & 84 & \\
S-1 & 7 & & & \\
\hline
\end{tabular}


tively. No grade 4 hematological toxicity was observed during either PTX or S-1 administration.

The most frequent nonhematological toxicity observed during PTX administration was alopecia (41 cases; $82 \%$; Table 5). Grade 2 alopecia resolved within 9 weeks following the last administration of PTX. One patient showed grade 3 neuropathy during the second course of PTX treatment, with grade 2 neuropathy persisting for 12 weeks following completion of PTX treatment.

During S-1 administration, alopecia remained the most frequent adverse event. In comparison to the occurrence during PTX administration, alopecia affected fewer patients, and those affected recovered. The number of patients with fatigue, and anorexia was greater during S-1 treatment than during PTX treatment, and hyperpigmentation was shown in nine patients during S1 treatment, whereas this was not seen during PTX treatment. Grade 3 nonhematological toxicity (diarrhea) was observed in one patient during S-1 treatment (Table 6).

\section{Discussion}

Despite considerable advances in treatment modalities for gastric cancer patients, the 5-year survival rate of resected T3 and T4 cancers in Japan is about 30\% [14]. Gastric cancer has a high tendency for recurrence as peritoneal dissemination $[15,16]$. The treatment choice is limited once peritoneal dissemination has occurred.

Table 3. Number of patients whose doses were decreased and treatment schedules were postponed

\begin{tabular}{lllllllll}
\hline & \multicolumn{3}{c}{ PTX } & & \multicolumn{5}{c}{ S-1 } \\
\cline { 2 - 4 } \cline { 6 - 8 } Course & 1 & 2 & 3 & & 4 & 5 & 6 & 7 \\
\hline Dose decreased & 0 & 2 & 3 & 4 & 4 & 1 & 1 \\
Postponed & 0 & 2 & 1 & 2 & 1 & 1 & 1
\end{tabular}

The Japanese Gastric Cancer Association issued the first version of the in gastric cancer treatment guidelines in March 2001. Nakajima [17] has presented an overview of these guidelines. The guidelines essentially define treatment indications according to the clinical stage of the cancer, as defined by the Japanese Gastric Cancer Association. For T3N0 cancers, clinical trials should be conducted to establish standard regimens for adjuvant chemotherapy. For T3N1 and T4N0 cancers, clinical trials of adjuvant and neoadjuvant chemotherapy are indicated. For T3N2 and T4N1 cancers, adjuvant chemotherapy, neoadjuvant chemotherapy, and adjuvant radiotherapy should be performed in the setting of ran-

Table 5. Nonhematological toxicities during PTX administration

\begin{tabular}{lrcc}
\hline Adverse reaction & Grade 1 & Grade 2 & Grade 3 \\
\hline Neuropathy & 10 & 0 & 1 \\
Myalgia & 3 & 0 & 0 \\
Lumbago & 2 & 0 & 0 \\
Back pain & 1 & 0 & 0 \\
Joint pain & 4 & 0 & 0 \\
Fatigue & 14 & 1 & 0 \\
Anorexia & 7 & 0 & 0 \\
Vomiting & 4 & 0 & 0 \\
Nausea & 7 & 0 & 0 \\
Stomatitis & 7 & 1 & 0 \\
Dysgeusia & 2 & 0 & 0 \\
Diarrhea & 9 & 0 & 0 \\
Alopecia & 23 & 18 & 0 \\
Weight gain & 2 & 0 & 0 \\
Weight loss & 3 & 0 & 0 \\
Edema & 1 & 0 & 0 \\
PS down & 1 & 0 & 0 \\
Fever & 2 & 0 & 0 \\
Skin rash & 5 & 0 & 0 \\
Rash & 2 & 0 & 0 \\
Cough & 1 & 0 & 0 \\
Genital bleeding & 1 & 0 & 0 \\
Allergy & 1 & 0 & 0 \\
Rhinitis & 1 & 0 & 0 \\
Hypotension & 1 & 0 & 0 \\
\hline PS perforance & & &
\end{tabular}

${ }^{\text {a } P S, ~ p e r f o r m a n c e ~ s t a t u s ~}$

Table 4. Hematological toxicities

\begin{tabular}{lrrrr}
\hline & Grade 1 & Grade 2 & Grade 3 & Grade 4 \\
\hline PTX $(n=50)$ & & & & \\
Leucopenia & 19 & 10 & 1 & 0 \\
Neutropenia & 16 & 9 & 4 & 0 \\
Thrombocytopenia & 3 & 0 & 0 & 0 \\
Anemia & 26 & 14 & 0 & 0 \\
S-1 $(n=46)$ & & & & 0 \\
Leucopenia & 12 & 12 & 0 & 0 \\
Neutropenia & 11 & 0 & 0 & 0 \\
Thrombocytopenia & 9 & 13 & 1 & 0 \\
Anemia & 25 & &
\end{tabular}


Table 6. Nonhematological toxicities during S-1 administration

\begin{tabular}{lrcc}
\hline Adverse reaction & Grade 1 & Grade 2 & Grade 3 \\
\hline Neuropathy & 6 & 1 & 0 \\
Myalgia & 2 & 0 & 0 \\
Joint pain & 1 & 0 & 0 \\
Fatigue & 17 & 2 & 0 \\
Anorexia & 13 & 7 & 0 \\
Vomiting & 4 & 0 & 0 \\
Nausea & 7 & 0 & 0 \\
Stomatitis & 6 & 0 & 0 \\
Dysgeusia & 1 & 0 & 0 \\
Diarrhea & 8 & 1 & 1 \\
Alopecia & 15 & 11 & 0 \\
Weight gain & 1 & 2 & 0 \\
Weight loss & 3 & 3 & 0 \\
Fever & 2 & 0 & 0 \\
Skin rash & 2 & 0 & 0 \\
Headache & 0 & 2 & 0 \\
Conjunctivitis & 2 & 0 & 0 \\
Hyperpigmentation & 9 & 0 & 0 \\
Dyspnea & 0 & 1 & 0 \\
Itching & 2 & 1 & 0 \\
Lung fibrosis & 1 & 0 & 0 \\
Excoriation & 0 & 1 & 0 \\
Photosensitivity & 1 & 0 & 0 \\
Vertigo & 1 & 0 & 0 \\
Hyposmia & 1 & 0 & 0 \\
Epigastralgia & 1 & 0 & 0 \\
Dying & & &
\end{tabular}

domized controlled trails. Thus, to summarize, there is no established adjuvant chemotherapy regimen for $\mathrm{T} 3$ or T4 cancers following resection with curative intent.

A unique approach, involving oral chemotherapy for postoperative adjuvant chemotherapy, has been used in Japan for a number of decades. Among these approaches UFT (tegafur and uracil) has been most widely used in both clinical trials and in general clinical practice. In recent years, UFT has been gradually replaced by S-1 (tegafur, 5-chloro-2, 4-dihydroxypyridine, and oxonic acid) treatment in general clinical practice, due to high response rates for this agent in two phase II studies in advanced cancers [18,19]. A phase III study to confirm the efficacy of S-1 treatment in an adjuvant setting is ongoing, and the final results are expected to be published in the year 2010 .

The results of a study carried out by Kitamura et al. [7] provided evidence that S-1 could provide promising treatment for patients with disseminated gastric cancer. Several reports suggest that systemic administration of PTX is effective for the control of malignant ascites in gastric cancer patients $[9,10]$. In two phase I studies of combination chemotherapy, using PTX + 5-fluorouracil (5-FU) and PTX + cisplatin (CDDP), we measured PTX levels in malignant ascites in two patients with advanced gastric cancer. One patient registered in the
PTX + 5-FU study and given $60 \mathrm{mg} / \mathrm{m}^{2}$ of PTX had serum PTX levels measured $60 \mathrm{~h}$ after PTX administration. The other patient, given $80 \mathrm{mg} / \mathrm{m}^{2}$ of PTX, had serum PTX levels measured $168 \mathrm{~h}$ after PTX administration. In these two patient's, the plasma levels of PTX remained within the effective range for 60 and $72 \mathrm{~h}$ respectively [20]. These results together with those cited above $[7,9,10]$, suggest that sequential chemotherapy using PTX and S-1 is promising for the prevention of peritoneal dissemination of T3/T4 advanced gastric cancer following R0 resection. There are, as yet, no established combination chemotherapy regimens for advanced gastric cancer. In the adjuvant setting after $\mathrm{R} 0$ resection, the target of chemotherapy is micrometastasis, in which cancer cells are heterogeneous, unlike those found in a bulky metastasis. Thus, in the present study, we used two agents for adjuvant chemotherapy, with PXT administration preceding S-1 administration 2 weeks after surgical treatment [21]. We carried out PTX administration before S-1 administration, both because intravenous administration is better than oral administration and to avoid the gastrointestinal toxicity often associated with $\mathrm{S}-1$, because the chemotherapy started from about 2 weeks after the operation. There is no evidence to suggest the appropriate duration of PTX administration in adjuvant chemotherapy for gastric cancer. Therefore, in the present study, we referred to the data of Henderson et al. [22], showing that sequential administration of PTX for 3 months after doxorubicin and cyclophosphamide administration improved the disease-free outcome and overall survival of breast cancer patients.

A randomized phase III trial with a two-by-two factorial design was planned in order to elucidate the survival benefit of the additional use of PTX administration compared with the administration of the two most popular oral fluoropyrimidines (UFT or S-1) alone. A comparison of UFT and S-1 was also included. The protocol for this study was approved by the Protocol Review Committee of the Japan South West Oncology Group (JaSWOG) [11]. Among the four treatment methods used in this phase III trial, PTX followed by S1 was predicted to be the most intense. For this reason, the feasibility of sequential chemotherapy using PTX and S-1 was investigated in the present study, and, in this study, the overall compliance rate with this treatment was $84 \%$, which is satisfactory even though patients started within 63 days after radical surgery. There were no patients presenting with grade 4 hematological toxicity. There were two patients with grade 3 nonhematological toxicity; in one of these patients, this toxicity occurred as grade 3 neuropathy in the second course of PTX administration. This patient showed grade 2 neuropathy in the following course of PTX treatment, and accomplished the complete treatment 
schedule; the duration of the grade 3 neuropathy was 4 weeks. The other patient presented with diarrhea in the second course of S-1 administration. This patient also presented with grade 1 diarrhea in the third course of S1 treatment; however, the patient did not present with diarrhea in the last course and could have accomplished the entire treatment schedule.

From our feasibility study, sequential PTX/S-1 chemotherapy is assumed to be safe and feasible as postoperative adjuvant chemotherapy. We have now commenced a randomized phase III trial with a two-bytwo factorial design to compare the sequential use of PTX and the oral fluoropyrimidines UFT and S- 1 to the use of these oral fluoropyrimidines alone.

Acknowledgments This work was supported in part by the Kyoto University EBM center and by the non-profit Epidemiological and Clinical Research Information network (ECRIN).

\section{References}

1. Yonemura Y, Fujimura T, Nishimu G, Falla R, Sawa T, Kataama $\mathrm{K}$, et al. Effects of intraoperative chemohyperthermia in patients with gastric cancer with peritoneal dissemination. Surgery 1996; 119:437-44

2. Hanazaki K, Mochizuki Y, Machida T, Yokoyama S, Sodeyama $\mathrm{H}$, Sode $\mathrm{Y}$, et al. Post-operative chemotherapy in non-curative gastrectomy for advanced gastric cancer. Hepatogastroenterology 1999;46:1238-43.

3. Bando E, Yonemura Y, Takeshita Y, Taniguchi K, Yasui T, Yoshimitsu Y, et al. Intraoperative lavage for cytological examination in 1297 patients with gastric carcinoma. Am J Surg 1999; 178:256-62.

4. Vogel P, Ruschoff J, Kummel S, Zirngibl H, Hofstadter F, Hohenberger W, et al. Prognostic value of microscopic peritoneal dissemination: comparison between colon and gastric cancer. Dis Colon Rectum 2000;43:92-100.

5. Osugi H, Takada N, Takemura M, Kaseno S, Lee S, Ueno M, et al. Oral fluroropyrimidine anticancer drug TS-1 for gastric cancer patients with peritoneal dissemination. Oncol Rep 2002; 9:811-5.

6. Kobayashi O, Konishi K, Kanari M, Cho H, Yoshikawa T, Tsuburaya A, et al. Unusual survival for more than 2 years with peritoneal metastases of gastric cancer. Gastric Cancer 2002;5:4750 .

7. Kitamura Y, Hayashi K, Sasagawa T, Oguma H, Takasaki K. Pilot study of S-1 in patients with disseminated gastric cancer. Drugs Exp Clin Res 2003;29:125-30.

8. Yonemori K, Shimada Y, Goto A, Ura T, Arai T, Hamaguchi T, et al. Retrospective analysis of clinical results and predictors of response in chemo-naïve patients with advanced gastric cancer treated with S-1, and oral fluoropyrimidine derivative, as singleagent chemotherapy. Gastric Cancer 2004;7:204-10.
9. Ishida T, Shimokawa H, Kawaguchi K, Nose N, Ikegami T, Itoh $\mathrm{H}$, et al. Effective weekly paclitaxel administration for gastric cancer with malignant ascites. A case report (in Japanese with English abstract). Gan to Kagakuyouhou (Jpn J Cancer Chemother) 2002;29:1643-6.

10. Cho H, Kanari M, Sano H, Tsuburaya A, Kobayashi O, Sairenji $\mathrm{M}$, et al. A case of metastatic gastric cancer responding to weekly administration of paclitaxel as a second-line therapy (in Japanese with English abstract). Gan to Kagakuyouhou (Jpn J Cancer Chemother) 2003;30:129-32.

11. Tsuburaya A, Sakamoto J, Morita S, Kodera Y, Kobayashi M, Miyashita Y, et al. A randomized phase III trial of postoperative adjuvant oral fluoropyrimidine vs sequential paclitaxel/oral fluoropyrimidine; and UFT vs S1 for T3/T4 gastric carcinoma: the Stomach Cancer Adjuvant Multi-institutional Trial Group (SAMIT) trial. Jpn J Clin Oncol 2005;35:672-5.

12. Oken MM, Creech RH, Tormey DC, Horton J, Davis TE, McFadden ET, et al. Toxicity and response criteria of the Eastern Cooperative Oncology Group. Am J Clin Oncol 1982;6:64955 .

13. National Cancer Institute: NCI common toxicity criteria, version 1. In: Investigators handbook: a manual of participants in clinical trials of investigational agents. Sponsored by the Division of Cancer Treatment, National Cancer Institute. Bethesda: MD, U.S. Department of Health and Human Services, Public Health Service, National Institute of Health; 1993.

14. Miwa Registry Institute for Gastric Cancer. Treatment results of gastric carcinoma in Japan (1979-1982). Tokyo: Japanese Research Society for Gastric Cancer, Mitamura Press; 1995.

15. Marrelli D, Roviello F, De Manzoni G, Morgagni P, Di Leo A, Saragoni L, et al. Italian Research Group for Gastric Cancer. Different pattern of recurrence in gastric cancer depending on Lauren histological type: longitudinal study. World J Surg 2002; 26:1160-5.

16. Fotia G, Marrelli D, De Stefano A, Pinto E, Roviello F. Factors influencing outcome in gastric cancer involving muscularis and subserosal layer. Eur J Surg Oncol 2004;30:930-4.

17. Nakajima T. Gastric cancer treatment guidelines in Japan. Gastric Cancer 2002;5:1-5.

18. Sugimachi K, Maehara Y, Horikoshi N, Shimada Y, Sakata Y, Mitachi Y, et al. An early phase II study of oral S-1, a newly developed 5-fluorouracil derivative for advanced and recurrent gastrointestinal cancers. Oncology 1999;57:202-10.

19. Sakata Y, Ohtsu A, Horikoshi K, Sugimachi K, Mitachi Y, Taguchi T. Late phase II study of novel oral fluoropyrimidine anticancer drug S-1 (1 M tegafur-0.4 M gimestat-1 M otastat potassium) in advanced gastric cancer patients. Eur J Cancer 1998;34: 1715-20.

20. Kobayashi M, Sakamoto J, Namikawa T, Okamoto K, Okabayashi T, Ichikawa K, et al. Pharmacokinetic study of paclitaxel in malignant ascites from advanced gastric cancer patient. World J Gastroenterol 2006;12:1412-5.

21. Klein CA, Blankenstein TJ, Schmidt-Kittler O, Petronio M, Polzer B, Stoecklein NH, et al. Genetic heterogeneity of single disseminated tumour cells in minimal residual cancer. Lancet 2002;360:683-9.

22. Henderson IC, Berry DA, Demetri GD, Cirrincione CT, Goldstein LJ, Martino S, et al. Improved outcomes from adding sequential paclitaxel but not from escalating doxorubicin dose in an adjuvant chemotherapy regimen for patients with nodepositive primary breast cancer. J Clin Oncol 2003;21:976-83. 